\title{
Interval Timing, Dopamine, and Motivation
}

\author{
Fuat Balcı * \\ Department of Psychology, Koç University, Rumelifeneri yolu, Sarıyer, Istanbul, Turkey \\ Received 7 April 2014; accepted 15 September 2014
}

\begin{abstract}
The dopamine clock hypothesis suggests that the dopamine level determines the speed of the hypothetical internal clock. However, dopaminergic function has also been implicated for motivation and thus the effect of dopaminergic manipulations on timing behavior might also be independently mediated by altered motivational state. Studies that investigated the effect of motivational manipulations on peak responding are reviewed in this paper. The majority of these studies show that a higher reward magnitude leads to a leftward shift, whereas reward devaluation leads to a rightward shift in the initiation of timed anticipatory behavior, typically in the absence of an effect on the timing of response termination. Similar behavioral effects are also present in a number of studies that investigated the effect of dopamine agonists and dopamine-related genetic factors on peak responding. These results can be readily accounted for by independent modulation of decision-thresholds for the initiation and termination of timed responding.
\end{abstract}

Keywords

Averaging artifact, clock speed, dopamine clock hypothesis, interval timing, motivation, peak interval procedure

\section{Introduction}

The ability to flexibly keep track of time intervals in the seconds to minutes range is observed in many vertebrate species with common statistical features (Buhusi \& Meck, 2005). This ability is referred to as interval timing. The alteration of interval timing behavior, as a result of different behavioral and neurobiological manipulations, has received a vast amount of attention in the study of human and animal time keeping ability. One influential line of research on this front has primarily focused on the role of dopamine in interval timing (for a review, see Coull et al., 2011). Generally speaking, these studies showed that timed responses can be sped up (shifted leftward) or slowed down (shifted rightward) by acutely increasing and

\footnotetext{
*E-mail: fbalci@ku.edu.tr
} 
decreasing dopaminergic tone in the brain, respectively (Çevik, 2003; Maricq \& Church, 1983; Maricq et al., 1981; Matell et al., 2006b; Meck, 1983, 1996). These findings have been canonically attributed to the effects of dopaminergic manipulations on the speed of the hypothetical internal clock and thus the change in the speed by which subjective time elapses (Meck, 1983,1996). On the other hand, dopamine also plays an important role in motivation (e.g., Berridge \& Robinson, 1998; for review see Berridge, 2007; Wise, 2004). Thus, at least in some cases (e.g., depending on the dose, dosing regime, level of training) the observed effects of dopaminergic manipulations on timing behavior might be mediated by the resultant changes in the motivational state of the organism being tested. This paper will re-evaluate the effects of dopaminergic manipulations on timing behavior as assessed on the peak interval (PI) procedure, and compare them to the effects of motivational manipulations with a specific emphasis on theory-driven analytical issues.

\subsection{Scalar Timing Theory (STT) and Peak Interval Procedure}

The PI procedure is a widely used task that has proved very instrumental in translational studies of the time keeping ability in humans and animals (Catania, 1970; Rakitin et al., 1998; Roberts, 1981). In this task, originally developed for animal research, subjects are initially trained on a discrete fixed interval (FI) schedule [composed of cycles of conditioned stimulus (CS) and inter-trial interval (ITI)]. In FI trials, the first response emitted after a pre-specified fixed CS duration is reinforced and terminates the CS. For instance, in an FI40s schedule, the first response after the 40th second of the CS (e.g., white noise stimulus) presentation would be reinforced while responses that were emitted during the first $40 \mathrm{~s}$ of the same CS or the ITI would not be reinforced or penalized.

When the response rates are calculated across multiple FI trials (e.g., average per each one-second bin), the resultant response curve would typically reveal a gradual increase in response rate as a function of trial time until the fixed interval. This response profile can be thought to reflect a gradual increase in reward expectancy as the elapsed time approaches to the availability of the reward (e.g., Gibbon, 1977). Importantly, the typical response pattern in single FI trials does not corroborate the gradual modulation of response rates observed in average FI response curves. At the single trial level, subjects typically do not respond (or respond at a low rate) during the first half of the fixed interval (on average until FI/2), and then start responding (or switch to high response rate) during the second half of this interval (Schneider, 1969). In other words, single trial based responding points at roughly a two-state system with an initial 'break' period (associated with a low rate of responding) followed by a 'run' period (associated with a high rate of responding).

The procedural censor, namely the delivery of the reinforcement and the cessation of the CS contingent upon the first post-FI response, does not enable capturing the timing behavior from this point onward without reinforcement/stimulusrelated contamination. The PI trials serve to fill this procedural gap and help cap- 
ture a more complete picture of timing behavior. In these probe (PI) trials, the CS lasts much longer than the FI schedule (e.g., PII20s for FI40s) and the reinforcement is omitted. Different trial types (FI or PI) are not signaled by a discriminative stimulus and thus subjects rely solely on the passage of time to guide their anticipatory responses.

When response rates are calculated across multiple PI trials, it typically yields a bell-shaped peak response curve with several characteristic features (e.g., Yi, 2007). Two of these properties are the most noteworthy with respect to the interval timing performance: (1) The global maximum (peak) of the response curve is typically located at around the FI schedule, suggesting that the maximal expectancy of reinforcement occurs at or around the reinforcement availability time (indexing temporal accuracy). (2) The variability of response times has a well-defined relationship to the mean within an individual; the standard deviation of response times is proportional to their mean (constant coefficient of variation, indexing temporal precision). The second feature points at the scale invariance of interval timing (Gibbon, 1977); when the peak response curves of an individual for different target intervals are expressed on a relative time scale [e.g., $t / \operatorname{mean}(t)]$, they superpose. These statistical features of peak responding are evident in data gathered from different species (e.g., Buhusi et al., 2009; Malapani \& Fairhurst, 2002; Rakitin et al., 1998).

As in the case of FI trials, the response patterns in individual PI trials do not fully corroborate the picture depicted by the average peak response curve. In individual PI trials, animals tend to initiate responding on average after the first half of the fixed interval (identically to FI trials) and when the post-FI responses are not reinforced, animals terminate responding in a fairly abrupt fashion (e.g., after $1.5 \times \mathrm{FI}$ ). Consequently, animals exhibit roughly a 'break-run-break' pattern in individual PI trials contrasting with the gradual change in average response rates (e.g., Cheng \& Westwood, 1993; Church et al., 1991, 1994; Gibbon \& Church, 1992). The trial time at which the animal initiates responding is referred to as the 'start time' and the trial time at which the animal terminates its ongoing responses is referred to as the 'stop time'. The scalar property can also be observed in the single-trial-based responses; spread of the run period (stop time-start time) tends to scale with the FI schedule. Figure 1 shows sample raster plots from representative individual rats (data from Church et al., 1998) for three different target intervals (FI30s, FI45s, FI60s). Each black dot corresponds to an individual operant response (i.e., lever press). The dots colored in green and red indicate the responses that mark the start time and stop time for the corresponding PI trial, respectively. As evident in these plots, the width of run period scales with the target interval even at the level of individual trials.

The interval timing behavior (including the abrupt transitions between low and high rates of responding in individual PI trials) has been nicely accounted for by the information-processing variant of the Scalar Expectancy Theory (or Scalar Timing Theory; Gibbon et al., 1984). According to this prominent model of interval 
timing, the clock signals are emitted continuously by a hypothetical pacemaker. The pacemaker is assumed to be linked to an accumulator via a switch mechanism. When attention is diverted to the event, the switch is closed (acting as an electronic switch) and the clock signals generated by the pacemaker start being integrated in the accumulator. When the event predicts a biologically crucial output (e.g., food pellet for a food-deprived animal or an aversive stimulus such as an electric shock), the accumulator-based magnitude representation of the current event duration is assumed to be consolidated in the reference memory. This longterm memory (LTM) representation of the event duration is updated after each experience of the same event. The primary source of scalar noise is assumed to originate from the process of transferring the representation of the event duration from the accumulator (or working memory) to the reference memory (e.g., consolidation noise). During this process, the accumulator-based values are assumed to be subject to Gaussian-distributed noise with a mean of 1 and a standard deviation of $\sigma$. Consequently, the resultant reference memory representation of the event duration can be conceptualized as an approximately normally distributed set of temporal values.

When the same event is experienced again, a random value is sampled from the reference memory and the accumulator-based representation of elapsing time is compared to this sample by a comparator mechanism in real time based on a ratio rule. In the context of the PI procedure, the comparator mechanism might for instance compute the following ratio, |Elapsed Time - LTM Sample|/LTM Sample. When this value crosses a decision threshold, the comparator would trigger responses in anticipation of the reinforcement until the same value crosses the same decision threshold once again. This comparison scheme can nicely account for the break-run-break pattern observed in the individual PI trials described earlier (see Fig. 1). Figure 2 illustrates the evolution of the temporal distance metric as a function of trial time in three different trials for a fixed target interval as well as the thresholds that are assumed to determine the initiation and termination of the timed anticipatory responding. The same figure also illustrates the predicted response windows (run period) in these three different trials. The temporal characteristics of the break-run-break pattern can be estimated from the empirical data in each trial by applying algorithms developed to detect state transitions (e.g., Balcı et al., 2009b; Cheng \& Westwood, 1993; Church et al., 1994; Gallistel et al., 2004a; Taylor et al., 2007), one of which was used to estimate start and stop times in Fig. 1. As can be observed in this illustration, due to the sources of noise (e.g., memory noise) in this process, the temporal characteristics of the break-run-break pattern exhibit substantial variability from one trial to the next. It is primarily due to this noise that when averaged across multiple PI trials, the resultant peak response curve does not preserve a boxcar profile but rather yields a smooth bell curve. Note that the timing noise can also be contributed by trial-to-trial variations in the pace- 

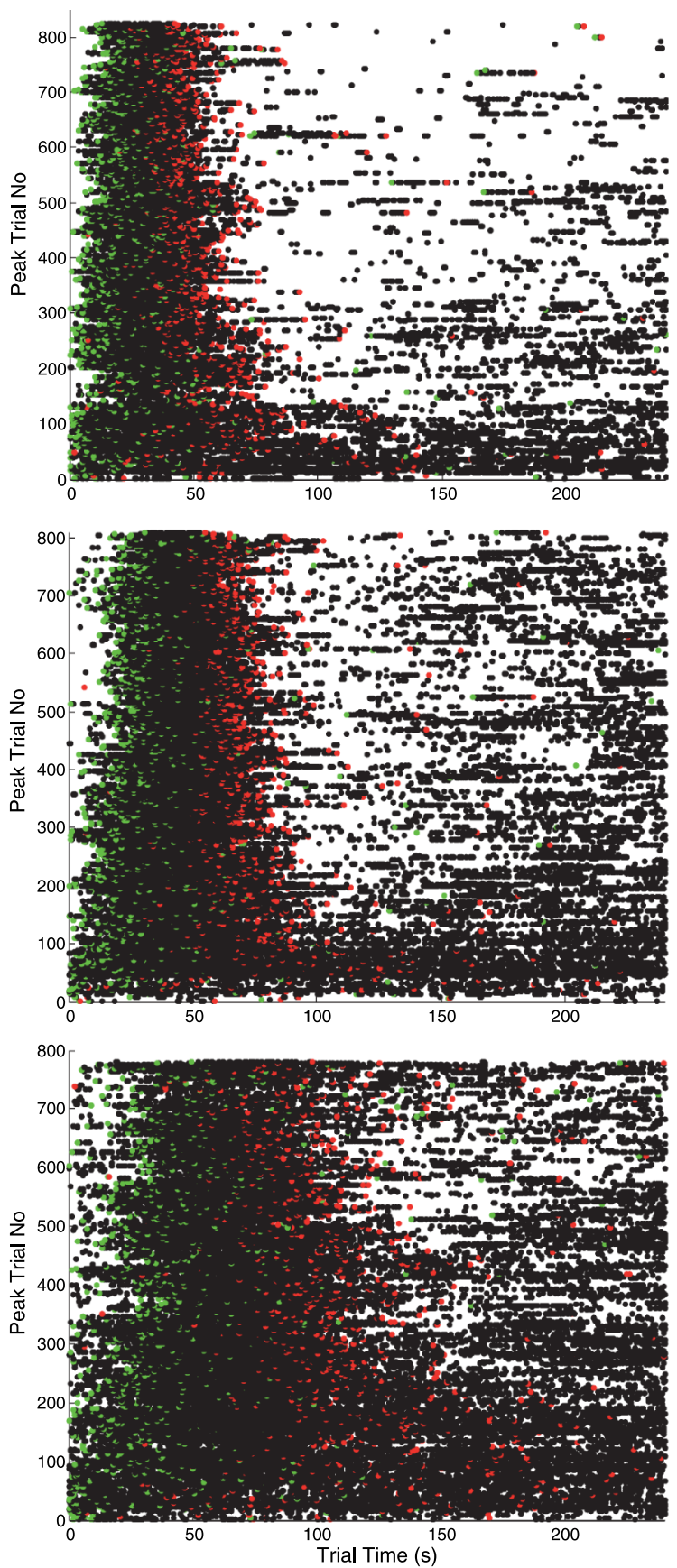

Figure 1. Sample raster plots from three representative rats trained on three different FI schedules (FI30s, FI45s, FI60s). Green dots indicate start times whereas the red dots indicate stop times for each PI trial. Data were provided graciously by Russell Church (Church et al., 1998). 


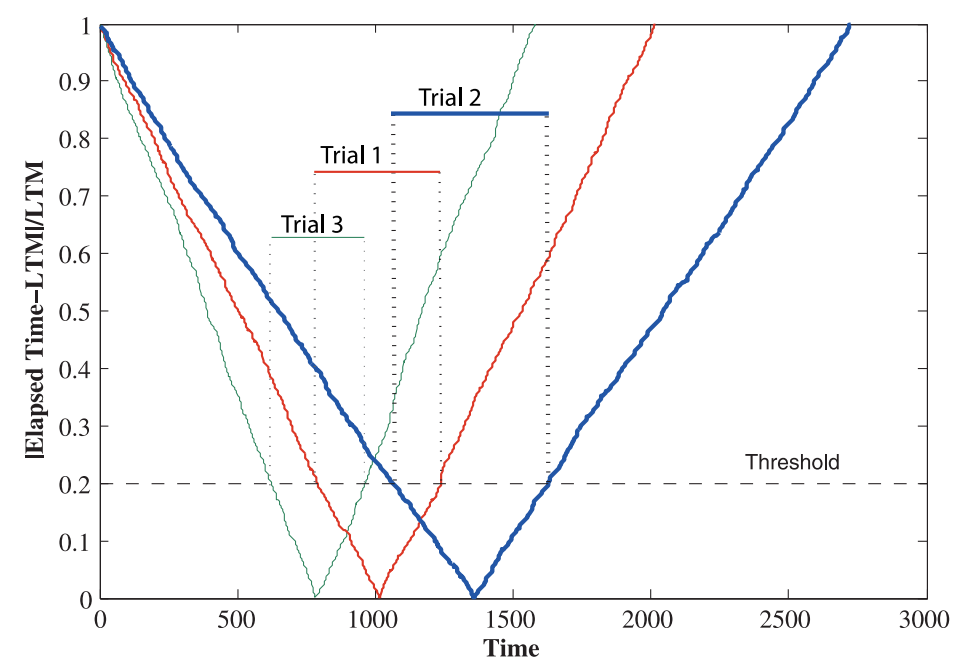

Figure 2. Illustration of the temporal decision-making (using a Poisson pacemaker) as it takes place in the PI trials. Illustrative data are shown for a given target interval (i.e., 1000 arbitrary time units) in three different trials, each with a different random LTM memory sample (referred to as LTM in the axis label). Each trial is indicated by a different line thickness. Dashed horizontal line shows the decision threshold, which when crossed initiates responses and when crossed again terminates responses. Solid horizontal lines represent the time windows over which the animal would respond (i.e., run period) in anticipation of the reinforcement in these three different PI trials. Note the dependence of the start and stop times on threshold crossings in different trials. This figure is published in color in the online version.

maker rate and/or decision thresholds. For simplicity, these sources of noise were set to zero in the simulations conducted as part of this work.

\subsection{Dopamine Clock Hypothesis}

The Scalar Timing Theory (STT) provides a powerful theoretic framework for the study of interval timing and the effect of different manipulations/factors on this function. For instance, one of the influential and inspiring lines of timing research have primarily relied on the STT framework in interpreting the effect of dopaminergic manipulations on timing behavior. A substantial number of these studies have shown that when dopamine levels were acutely increased (e.g., by injecting cocaine) in animals already trained on the PI procedure, their peak time shifted leftward as if the time passed faster for them under drug influence (Çevik, 2003; Maricq et al., 1981; Matell et al., 2006b; Meck, 1983). In these cases, the peak time was shorter than the target interval. Conversely, when dopamine levels were decreased acutely (e.g., by injecting haloperidol), the peak location of the average response curves shifted rightward as if the time passed slower for the animals under drug influence (Drew et al., 2003; Meck, 1983, 1986). In these cases, the peak time 
was longer than the target interval. Importantly, these shifts were proportional to the target interval (e.g., $0.11 \mathrm{FI}$ ).

These proportional shifts in peak time were originally interpreted within the STT framework; they were attributed to the effect of dopamine manipulations on the rate of the pacemaker, which constitutes the clock stage of the STT model. Specifically, under the dopamine clock hypothesis (Meck, 1983, 1996), higher levels of dopamine were assumed to increase, whereas lower levels of dopamine were assumed to decrease, the rate at which the hypothetical pacemaker emits the clock signals. Since the sufficient number of clock signals that code for a given time interval at the baseline condition can be accrued earlier with a faster clock, timed responses would also occur earlier under dopamine agonists. The opposite holds for the effect of dopamine antagonists.

Furthermore, the dopamine clock hypothesis nicely explains the re-normalization of response times (e.g., peak time) under chronic dopamine treatments again within the STT framework (Meck, 1983, 1996). As the event time is repeatedly experienced, the LTM representation of the fixed event duration would be updated according to the new clock speed and thus the mismatch between the current clock speed (under drug influence) and the clock speed 'assumed' in the LTM representation would gradually disappear. If the chronic dopamine treatment is stopped, then one observes shifts in timed responses in the opposite direction (e.g., rightward shift after stopping chronic dopamine agonist treatment; Meck, 1983, 1996).

Figure 3 illustrates how the clock speed effects would be manifested in individual trial-based peak responding within the STT framework. The top panel of Fig. 3 shows the behavior of the model in twenty independent trials with baseline dopamine levels (e.g., vehicle injection). In the bottom panel of the same figure, the Poisson pacemaker rate was increased by $10 \%$. Visual inspection of Fig. 3 suggests that when the clock speed is increased, the anticipatory responses should, on average, start and stop earlier (to the same degree) compared to the baseline.

In order to demonstrate the reflections of these effects in individual trials (Fig. 3) on the average peak response curves, the model was simulated for 5000 trials. When these simulated response data following the break-run-break pattern in individual trials were averaged across many PI trials, they yielded Gaussian-like response curves (Fig. 4). The solid curves in Fig. 4 illustrate the resultant peak response curves under baseline conditions for two different target times (1000 and 2000 arbitrary time units). The dashed curves in the top panel of Fig. 4 show the data after the clock speed was increased by $10 \%$ (as would be expected after dopamine agonist administration) whereas the dashed curves in the bottom panel of Fig. 4 show the data after the clock speed was decreased by 10\% (as would be expected after dopamine antagonist administration). Comparison of these curves points at overall proportional leftward and rightward shifts in the response curves and thus the peak time after an increase and decrease in the clock speed, respec- 

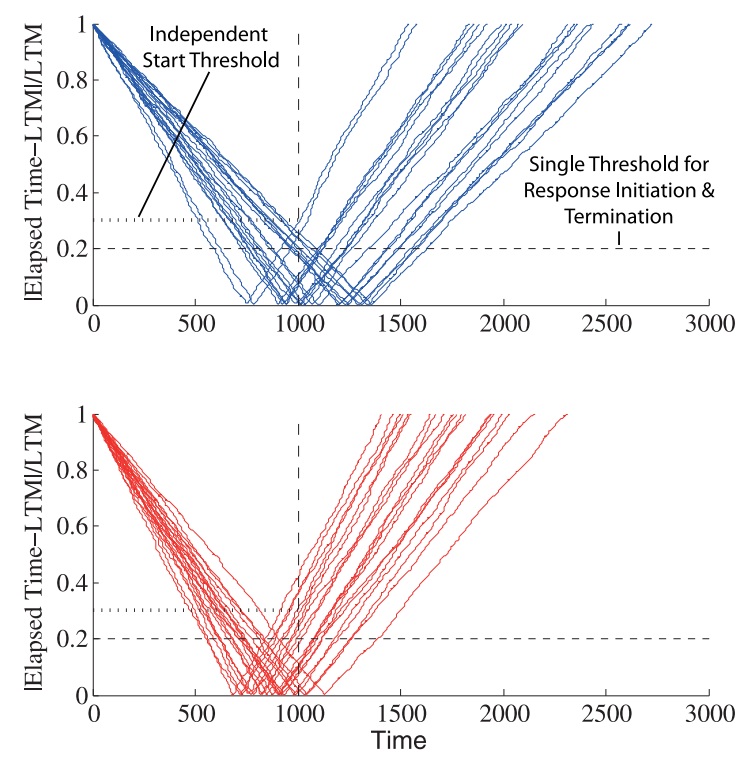

Figure 3. Top panel: Evolution of the temporal distance metric as a function of trial time for twenty different trials (e.g., under baseline conditions). Bottom panel: Evolution of the same metric after the clock speed was increased by $10 \%$ (e.g., after dopamine agonist administration). Dashed horizontal line is the decision threshold utilized for both response initiation and termination. Dotted horizontal line is the decision threshold assumed for response initiation only. Note that in the latter case, the thresholds for initiation and termination of anticipatory responses are independent (e.g., Balcı et al., 2009b; Church et al., 1994; Gallistel et al., 2004b). This figure is published in color in the online version.

tively. The proportional shifts in the peak times are indeed one of the widely used measures of the presumed clock speed effects (e.g., Cheng et al., 2007b) and this effect is clearly observed in Fig. 4.

The dopamine clock hypothesis has received substantive empirical support particularly from animal studies (Çevik, 2003; Maricq \& Church, 1983; Maricq et al., 1981; Matell et al., 2006b; Meck, 1983, 1996; for a review see Cheng et al., 2007b). Human data have revealed differential effects (e.g., Malapani et al., 2002; Malapani \& Rakitin, 2003; Rammsayer, 1989, 1993, 1997; Rammsayer \& Vogel, 1992; but see Lake \& Meck, 2013). However, it is possible that dopaminergic manipulations exert their effect on timing behavior in the PI procedure also in ways different from the presumed clock speed modulation. For instance, dopaminergic manipulations are known to affect the motivational state of animals (e.g., Berridge \& Robinson, 1998; Wise, 2004); dopamine activation enhances incentive salience (wanting; Berridge, 2007). Thus, alterations in the motivational state might be another independent potential source of an effect of dopaminergic manipulations on timing behavior. 

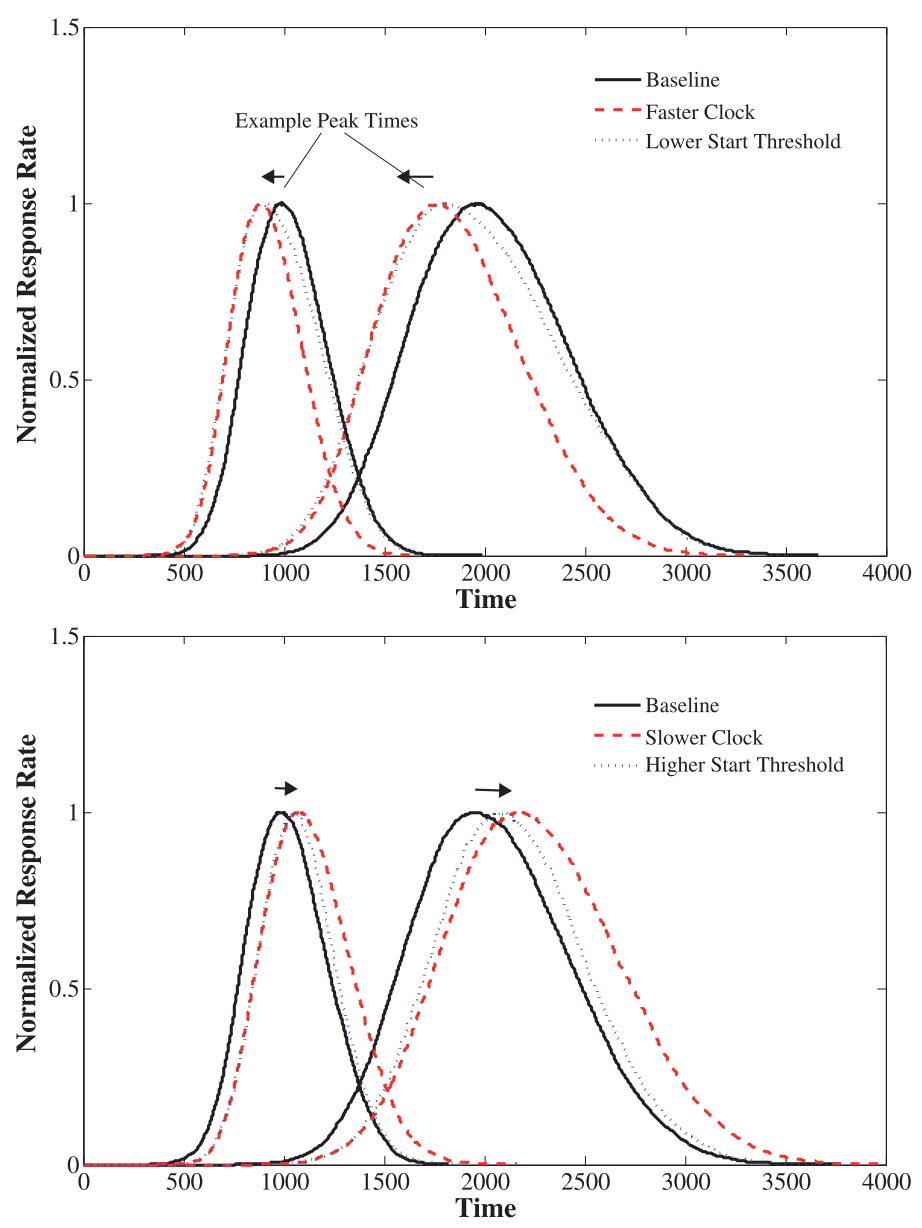

Figure 4. Solid curves show the average peak response curves calculated from simulated data for two different target intervals (i.e., 1000 and 2000 arbitrary time units) in the baseline conditions. Top panel: Dashed curves show the average peak response curves after the clock speed was increased by $10 \%$. Dotted curves show the simulated average response curves when the clock speed was kept constant but the start threshold was lowered (farther from the target interval) by a constant amount without changing the stop thresholds (note that the temporal distance metric is a ratio). Bottom panel: Dashed curves show the average peak response curves after the clock speed was decreased by $10 \%$. Dotted curves show the simulated average response curves when the clock speed was kept constant but the start threshold was increased (closer to the target interval) by a constant amount without changing the stop thresholds. The behavioral effects of threshold modulation will be addressed later in the paper. This figure is published in color in the online version.

\subsection{Motivation and Timing Behavior}

How can motivational state affect timing behavior? It might alter the speed of the hypothetical internal clock, modulate the decision threshold(s) or affect both the 
clock speed and decision threshold(s). Regarding the clock speed effects, higher incentive motivation might increase, whereas lower incentive motivation might decrease, the speed of the internal clock. In this case, acute motivational manipulations would be expected to lead to proportional shifts in start and stop times in the same direction and degree, and these effects should normalize under chronic motivational treatments. Regarding the decision threshold effects, higher incentive motivation might lower the start threshold or lower both the start and stop thresholds. These differential effects at the psycho-mechanistic level would have different predictions regarding the resultant timing behavior. Lowering start thresholds would lead to earlier start times but would not alter the stop times. If start and stop thresholds are both lowered (say due to their mechanistic dependence) on the other hand, start times would occur earlier and stop times would occur later. In any case, different from the clock speed effects, the threshold-dependent effects would not be expected to normalize after chronic alteration in the motivational state. To this end, a review of the literature regarding the effect of motivational manipulations on timing behavior would be informative. In this section, this literature will be reviewed and then the results from these studies will be evaluated in relation to the results regarding the dopaminergic modulation of timing behavior.

Early studies that investigated the effect of motivation on timing behavior have primarily based their analyses on the average peak response curves using the peak time as the critical unit of analysis. In this sense, these early studies resemble the majority of the experiments that investigated the role of dopamine in interval timing. In one of these early studies, Roberts (1981) tested rats on the PI procedure using a FI40s schedule and compared their performance between sessions with food deprivation $v$ s. pre-feeding prior to testing. These conditions were alternated within each successive pairs of sessions for each rat for 20 sessions. Based on the analysis of the average response curves, Roberts observed a rightward shift in the peak times after pre-feeding compared to the food deprivation condition but these effects held only for the initial test sessions. These shifts in the peak time would be typically attributed to a decrease in clock speed after pre-feeding (at least early in testing). On the other hand, since the effect of pre-feeding on peak time disappeared with further testing in this study, motivation was assumed not to affect the temporal characteristics of peak responding and to exert its primary effect on the peak rate (i.e., maximum response rate).

In another study, Grace and Nevin (2000) tested pigeons on the peak procedure (FI20s) with two different reward magnitudes (1.5 s or $4.5 \mathrm{~s}$ access to grain). Different reward magnitudes were altered between trials and they were signaled with visual stimulus of different colors. Grace and Nevin observed that pigeons exhibited later peak times when expecting the smaller reward magnitude (see also Kacelnik \& Brunner, 2002, for similar results with starlings). In another pigeon study, Plowright et al. (2000) investigated the effect of motivation on timing behavior by using pre-feeding manipulation. As in earlier studies, Plowright et al. also 
reported a rightward shift in the peak time. Finally, MacEven and Killeen (1991) manipulated the amount of access to grain between different test conditions $(1.5 \mathrm{~s}$, $3 \mathrm{~s}, 7 \mathrm{~s}$ ) and did not observe any significant effect on the temporal characteristics of peak responding (see also Hatten \& Shull, 1983).

Since the analyses conducted in these earlier studies were primarily based on the average response curves, the STT-driven mechanistic evaluation of the motivational effects on timed responding is difficult. Fortunately, a recent line of work that investigated the effect of motivation on peak responding used more elaborate analytical approaches (i.e., single trial response-based analyses) that were better suited for the psycho-mechanistic evaluations of the timing behavior. Several of these more recent studies primarily focused on the effect of different reward magnitudes on timing behavior (e.g., Galtress et al., 2009, 2010, 2012a; Ludvig et al., 2007,2011 ) whereas others investigated the effect of reward devaluation (e.g., prefeeding lithium chloride; see Galtress et al., 2009, 2012b).

The majority of these more recent studies revealed asymmetrical modulation of start times and stop times as a result of motivational manipulations. This was evident even in one of the earlier studies reviewed above; the visual inspection of Fig. $2 \mathrm{~b}$ in Plowright et al. (2000) suggests that the effect of pre-feeding was specific to start times (i.e., later start time after pre-feeding) without any apparent effect on stop times. In one of the more recent studies, Ludvig et al. (2007) investigated the effect of brain stimulation reward (BSR) intensity on peak responding (FI20s) by changing the BSR intensity in a chronic fashion between different test phases. When Ludvig et al. based their analysis on average peak response curves, as in the case of earlier studies, they detected a rightward peak time shift in the low BSR intensity condition. This result was consistent with the findings of earlier studies that used a similar approach (Grace \& Nevin, 2000; Plowright et al., 2000; Roberts, 1981) and it could be interpreted in terms of a change in clock speed (although chronic manipulation would nullify this interpretation when considered within STT). Interestingly however, the analysis of single-trial-based responses revealed a different picture; the rats started to respond later when the BSR intensity was reduced but they did not exhibit any change in their stop times as a result of this manipulation. This finding contradicted the interpretations based on a presumed overall shift in peak response curves and instead pointed at the modulation (i.e., reduction) of primarily the decision threshold to initiate timed anticipatory responding.

Galtress et al. (2012a) observed a very similar behavioral signature in their study. They tested rats with an FI60s schedule and three different levels of reward magnitude (one pellet, two pellets, and four pellets in different sessions-long tests). Galtress et al. observed that when the reward magnitude was increased, the start times occurred earlier without any change in stop times (although the order of manipulation seems to be an important factor - compare their Experiments 2 and 3). Similar observations were reported in an earlier study by the same researchers. Galtress and Kirkpatrick (2009) tested the effect of reward magnitude 
on peak responding (with FI60s) in rats over three phases. One group of rats received the 1-4-1 (pellet) reward magnitudes whereas the other group received the 4-1-4 (pellet) reward magnitudes between three different test phases. Thus, for the first group of rats the reward magnitude was first increased and then decreased between successive test phases whereas the reverse order held for the second group of rats. Galtress et al. observed that shifts from low (i.e., one pellet) to high (i.e., four pellets) reward magnitudes resulted in earlier start times whereas shifts from high to low reward magnitudes resulted in later start times (within group comparisons). The same differences between the start times were also observed in two independent between-group comparisons during the second and third phases of testing (i.e., one pellet $v s$. four pellets conditions). Importantly and consistent with other recent studies described above, these manipulations did not have any significant effect on the stop times.

Symmetrical effects on start times and stop times were however reported in another study by Galtress and Kirkpatrick (2010). They tested rats on the peak procedure (FI60s) with two different reward magnitudes between different phases (initially with one pellet and then with four pellets). Their analysis of timed responses at the level of individual trials revealed significant shortening of both start times and stop times in the high reward magnitude condition. That said, the visual inspection of the average peak response curves (their Fig. 8, top panel sham group data) did not reveal earlier peak times with the higher reward magnitude, which would be expected given the results of individual trial analyses. If anything, the observed patterns in this figure pointed at later peak times in the high reward magnitude condition. This discrepancy between the response profiles revealed in single trial based responding and the average response curves makes the interpretation of their findings difficult.

In this new line of studies described above, the reward magnitude manipulations were realized and tested primarily in a chronic fashion. Thus, within the STT framework, these effects cannot be attributed to the effect of motivation on clock speed, as the LTM representation of the target time interval would be assumed to normalize with repeated experience of the same FI schedule. A number of studies that have also adopted the individual trial-based analyses investigated the effect of motivational state on peak responding by changing reward magnitudes between trials and signaling them by discriminative stimuli. For instance, Ludvig et al. (2011) trained pigeons on an FI5s schedule with three different visual stimuli each signaling a different reward magnitude $(1,3$, and $9 \mathrm{~s}$ access to grain). In the majority of the trials, only one stimulus was presented and either the FI trial or the PI trial was in effect. In $20 \%$ of the FI trials, two stimuli were presented simultaneously and the pigeons were induced to make a choice, which determined the reward magnitude that would be in effect in that FI trial. In the PI trials, pigeons exhibited a behavioral signature in response to stimuli signaling different reward magnitudes that was very similar to the one observed in earlier studies. Pigeons started responding 
earlier when presented with the stimulus that predicted larger rewards; however, their stop times remained the same. The preferences of pigeons in choice trials validated the presumed effect of the motivational manipulation; as expected, the pigeons chose the stimuli that predicted larger reward magnitude.

Balcr et al. (2013) conducted a similar study with humans using a variant of the PI procedure. In their study, human participants were tested with two different FI schedules (FI10s and FI17s tested in different sessions) and with different reward magnitudes that changed randomly between different test blocks. The human participants started their timed responses proportionally earlier (proportional to the FI schedule) when expecting a larger reward magnitude whereas the timing of their response termination did not differ between different reward magnitude conditions.

The studies described above investigated the effect of motivational state on peak responding by changing the reward magnitudes. Another set of studies that have also adopted a single-trial-based response analysis manipulated the motivational state by devaluating reward with pre-feeding the animals or administring lithium chloride. For instance, Galtress et al. (2012b) investigated the effect of motivation on peak responding by pre-feeding rats prior to testing. In one of their experiments (Experiment 2), Galtress et al. (2012b) tested rats with two different FI schedules (FI30s and FI60s) and with two different rewards (not discussed here). The rats were trained while food-deprived whereas they were tested while pre-fed. Consistent with the majority of studies reported earlier, this experiment showed that regardless of the devalued reward type, the effect of pre-feeding was primarily on the start times. After pre-feeding rats delayed their start times compared to baseline and this effect was more pronounced in the FI60s schedule.

In another experiment (Experiment 1) by Galtress et al. (2012b), one group of rats was trained while food-deprived whereas the second group of rats was trained pre-fed. During the test phase of this experiment, those rats that were fooddeprived during training were tested pre-fed and rats that were trained pre-fed were tested after food deprivation. What Galtress et al. observed was interesting. Both groups had later start times during testing compared to training without any significant effect on stop times. In this study testing was conducted with PI trials only, which might have been the dominant factor that affected the start times in the same way in both groups although the results from extinction training did not yield such effects (see Experiment 3 of Galtress \& Kirkpatrick, 2009). The effect of pre-feeding specifically on start times (i.e., later start times) was also reported in a recent study conducted with pigeons (Fox \& Kyonka, 2014, FI15s).

Galtress and Kirkpatrick (2009) also tested the effect of reward devaluation on peak responding by LiCl administration (second part of Experiment 1, FI60s). This manipulation resulted in a rightward shift in both start and stop times different from the observed effects of reward magnitude in the first part of the same experiment (where the effect was only on start times). In the second experiment, 
Galtress and Kirkpatrick (2009) aimed to test the effect of transient manipulations of motivational state by pre-feeding the animals prior to testing (as opposed to manipulations presumably with long-lasting effects such as $\mathrm{LiCl}$ ). They first tested rats in an FI60s schedule and observed that pre-feeding and devaluation by $\mathrm{LiCl}$ resulted in a rightward shift in start times early in training without any effects on the stop times. They then tested the effect of pre-feeding with FI30s schedule in the same animals and observed that both start and stop times shifted rightward. The authors attributed these differences in the effect of motivation to the differential degrees of prior training. This assumption needs to be tested with further experiments.

Finally in a study conducted with mice, Balcr et al. (2010b) investigated the modulation of peak responding over the course of test sessions under the assumption that mice satiated over time with pellets received in FI trials. Consistent with earlier studies that conducted response curve based analysis, their analysis revealed a rightward shift in peak times as the session progressed. On the other hand, their single-trial-based analysis revealed that shifts in peak times were due to later start times in the absence of an effect on stop times.

The majority of these recent findings from pigeons, rats, mice, and humans suggest that motivational manipulations have a specific effect on the timing of the initiation of anticipatory responding; higher motivation leads to roughly proportional earlier start times. These effects were mostly observed in the absence of apparent changes in the timing of the termination of anticipatory responding (i.e., stop times). As had been demonstrated in several of these studies (e.g., Balcr et al., 2010; Ludvig et al., 2007), when responses were averaged between multiple PI trials in which animals exhibited earlier or later start times only, the resultant peak response curve could reveal a leftward and rightward shift in peak times, respectively.

Simulations were conducted in order to better demonstrate this potential averaging artifact. In these simulations, the speed of a Poisson clock was kept constant; however, the threshold for the initiation of responding was decreased or increased by a constant value without modifying the threshold for terminating responses. Simulated data were then averaged across multiple PI trials to calculate the average response curves (dotted lines; Fig. 4). Visual inspection of these response curves in Fig. 4 shows that just earlier and later start times (without a change in stop times) can lead to perfectly proportional leftward and rightward shifts in the peak times, respectively.

These findings might also explain the effect of reward magnitude in other timing paradigms such as the differential reinforcement of low rate (DRL) task. In the DRL task, subjects are trained to wait for a minimum amount of time before responding. Doughty and Richards (2002) tested both rats and pigeons on the DRL task with multiple schedules while manipulating the reward magnitudes in a chronic fashion (over weeks of testing). They observed that both rats and pigeons responded 
earlier when expecting a larger reward and these effects held over multiple test sessions. Note that in the DRL task a single critical timed response is emitted and this form of responding likely relies on behavioral mechanisms that underlie response initiation in the PI procedure.

\subsection{Dopamine and Interval Timing: Challenging Findings}

We will now get back to the effect of dopaminergic manipulations on peak responding. As discussed earlier, it is possible that at least in some cases the effects of dopaminergic manipulations on peak responding are mediated through their effect on the motivational state of the animals. Consequently and in the light of studies reviewed in the previous section, in these cases, what appears to be leftward and rightward shifts in the peak times as a result of dopaminergic manipulations impacting clock speed might in fact result from changes in the timing of response initiations due to alterations in the motivational arousal of the animal. A number of studies indeed supported this possibility. Remember that a clock speed effect would dictate the modulation of both start and stop times in the same direction and to the same degree as a result of acute dopaminergic manipulations and the effects are expected to normalize with chronic treatment.

Taylor et al. (2007) tested the effect of $0.5 \mathrm{mg} / \mathrm{kg}$ and $1 \mathrm{mg} / \mathrm{kg}$ D-amphetamine on timed responding in the PI procedure (FI24s) and they analyzed the data using two different methods. Their first analysis was based on the Gaussian curve fitting to average peak response curves for estimating the peak time. Their second set of analyses estimated the start and stop times from responses in individual PI trials. Taylor et al. showed that when the analyses were based on fits to average response curves, one would conclude that D-amphetamine administration resulted in a dose-dependent leftward shift in the peak location, which would be canonically attributed to a dose-dependent increase in clock speed. On the other hand, when the same data were evaluated at the level of individual trials, the leftward shift in timed responses appeared to be specific to start times (no effect on stop times). Then, Taylor et al. subtracted four seconds from the start times estimated from the empirical data without altering the stop times. Consistent with the current simulation work (Fig. 4), when the response curve fits were applied to the average of these biased data, it revealed a leftward shift in the peak time.

In another study, Gooch et al. (2007) tested the effect of methamphetamine on peak responding (FII5s) at three different doses (acute $0.5 \mathrm{mg} / \mathrm{kg}, 1.0 \mathrm{mg} / \mathrm{kg}$, $1.5 \mathrm{mg} / \mathrm{kg}$ ). Although they observed a leftward shift in the peak time, their singletrial analyses revealed a significant leftward shift only in the start times. They also argued that their findings were consistent with the asymmetrical effects of dopaminergic manipulations on the threshold setting. Similarly, Saulsgiver et al. (2006) showed that D-amphetamine administration in pigeons increased the response rate prior to reward availability time (pre-peak period) without affecting the response rates after the reward availability time. Importantly, this asymmet- 
rical effect on response rates led to an apparent leftward shift in the peak times, which was confirmed by simulations. Dopamine agonists are also known to shift the inter-response time distributions to the left in the DRL procedure (e.g., Paterson et al., 2011 - session-long testing) and as argued earlier, point-timed responses emitted in the DRL task might be underlain by the same dynamics that underlie response initiation in the PI procedure.

These results overall suggest that in the context of the studies discussed above, the effect of dopaminergic manipulations on peak times might be due to their modulation specifically of start times and importantly this behavioral signature resembles the effect of motivational manipulations in the same paradigm (note however sessions-long testing in these studies). The possible relationship between dopamine and motivation as it relates to timing performance was further supported by a study conducted with hyperdopaminergic dopamine transporter knockdown mice (DAT KD, Balcı et al., 2010). DAT KD mice under express dopamine transporters by $90 \%$ compared to wild type mice and consequently have chronically higher levels of tonic dopamine levels by $70 \%$ (Zhuang et al., 2001). DAT KD mice are known to have enhanced incentive motivation compared with the wild type mice without any differences in the rate of learning (Cagniard et al., 2006; Peciña et al., 2003; Yin et al., 2006). Balcı et al. (2010a) tested DAT KD mice in a dual peak procedure with two different FI schedules (FI30s and FI45s). One of the levers was associated with the short and the other lever was associated with the long FI schedule and different schedules were in effect during different periods of a test session. Balcı et al. observed that DAT KD mice had earlier start times compared to the WT mice however there was no difference between the groups in terms of their stop times. Consistent with earlier studies, when the analyses were based on the average response curves, DAT KD mice exhibited earlier peak times.

Since higher dopamine levels constitute a chronic condition in these mice, one would not expect a peak time shift based on clock speed effect however this effect was clearly present in these mice. In order to test the effect of dopamine antagonists, Balcı et al. also administered raclopride (a D2 receptor antagonist) to DAT KD and wild type mice (D2 receptor affinity has been shown to be critical for the clock speed effects; Meck, 1986). They observed that raclopride administration delayed the start times (more pronounced in DAT KD mice) and decreased the response rates in a dose-dependent manner. Interestingly however, raclopride did not have any effect on stop times. Note that the dopamine clock hypothesis would predict a rightward shift in both start times and stop times as a result of raclopride administration although no difference between the groups would be predicted for the baseline conditions.

Balcı et al.'s (2013) study with humans also pointed at the importance of dopamine related gene polymorphisms for the effect of motivational manipulations on peak responding. They found that participants with the combinations of COMT and DRD2 gene polymorphisms that are known to have increased reward 
sensitivity and enhanced approach behavior (Reuter et al., 2006) were also those that exhibited earlier start times when expecting a larger reward magnitude (note the lack of difference for stop times).

Some of the earlier studies that presented the average response curves also suggested that the effect of methamphetamine was more pronounced for start times (sometimes with no apparent effect on stop times). For instance, Fig. 4 of Maricq et al. (1981) — after visually normalizing by the maximum response rate - shows that the pre-peak period was widened without much effect on the post-peak period (see also Fig. 2 of Gershman et al., 2014 for the illustration of the same data). The same profile was also observed to a certain extent in Matell et al. (2004) especially for 10 and $30 \mathrm{~s}$ target intervals. Despite these findings that point at the primary sensitivity of start times to dopaminergic manipulations, Matell et al. (2006b) observed that the administration of methamphetamine specifically shifted the stop times to the left (FI10s \& FI30s) without any modulation of the start times. Abner et al. (2001) reported similar findings regarding the effect of D-amphetamine and methylphenidate on peak responding. These finding are challenging not only for the dopamine clock hypothesis but also for the motivation-based interpretations of start time modulation formulated in this paper. Table 1 summarizes the major findings from studies reviewed in the paper.

\section{Motivation and Temporal Discrimination}

The previous sections primarily discussed the effect of dopaminergic and motivational manipulations on timed anticipatory responding as assessed in the PI procedure. The effects of these factors have also been demonstrated using other interval timing paradigms such as the temporal bisection task (e.g., Maricq \& Church, 1983; Penney et al., 2008; Ward \& Odum, 2006, 2007). In the temporal bisection task, animals are initially trained to discriminate two reference durations that are signaled by the presentation durations of a stimulus (e.g., 3 vs. $6 \mathrm{~s}$ long tone). Specifically, animals are trained to emit one type of response (e.g., left lever press) after the presentation of the stimulus for a short duration and another type of response (e.g., right lever press) after the presentation of the same stimulus for a long duration.

After the discrimination performance reaches a pre-determined level (e.g., 80\% correct), the intermediate durations are presented intermixed with the reference durations and animals are induced to categorize each signal duration as short or long, presumably, based on the subjective similarity of the experienced duration to the reference durations. Only, the correct categorizations of the reference durations are reinforced. The observed proportion of the long responses typically has a sigmoidal relation (e.g., cumulative Gaussian curve) to the signal durations. The signal duration for which the probability of long categorization is 0.5 (e.g., the mean of the cumulative Gaussian fit) is referred to as the point of subjective equality (PSE). This duration is assumed to be subjectively equidistant to the short and 


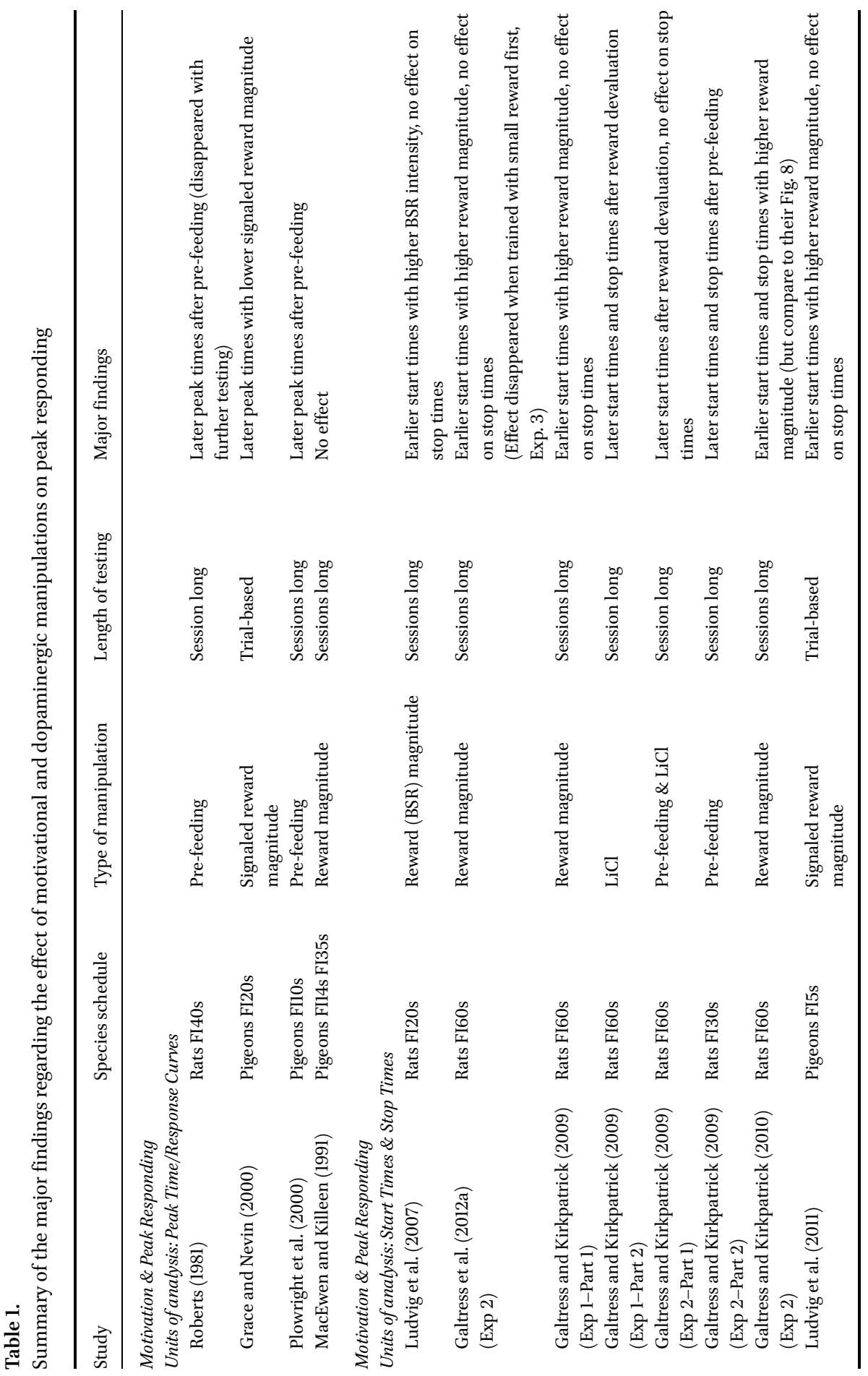




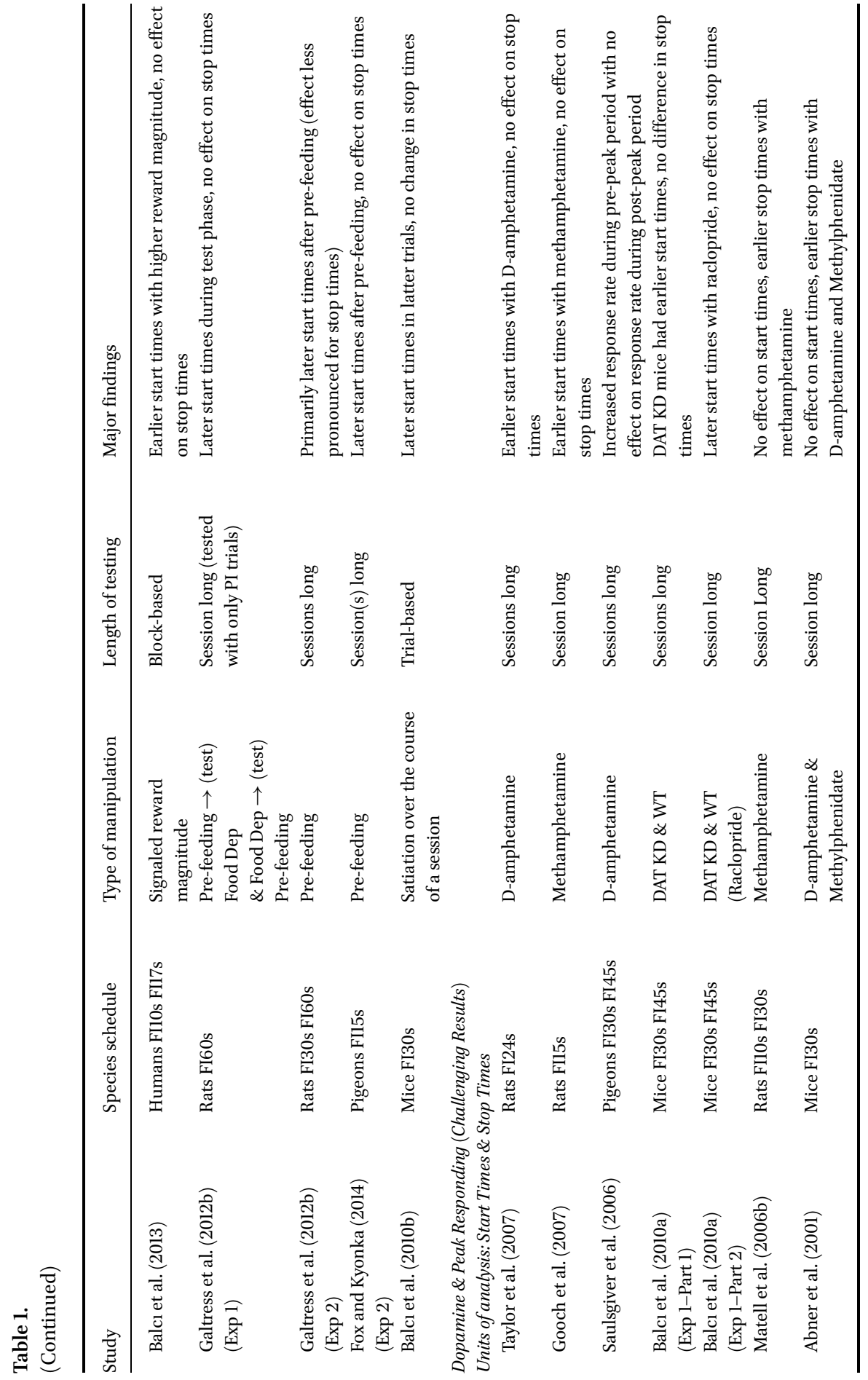


long reference durations. The slope of this curve (e.g., standard deviation of the cumulative Gaussian fit) on the other hand indexes the level of timing noise.

A number of studies have shown that dopamine agonists such as methamphetamine shifted the psychometric function to the left whereas dopamine antagonists such as haloperidol shifted the psychometric function rightward (e.g., Bizot, 1997; Çevik, 2003; Maricq \& Church, 1983; Meck, 1983, 1986, 1988). These effects have been consistently interpreted in terms of the effect of these pharmacological agents on the clock speed. On the other hand, several other studies have shown that dopamine agonists could also disrupt the overall temporal discrimination performance by flattening the temporal choice functions (e.g., Çevik, 2003; Chiang et al., 2000; McClure et al., 2005; Odum \& Ward, 2007; Odum et al., 2002; Santi et al., 1995). The latter findings were typically interpreted in terms of the disruption of attention to time or stimulus control by dopaminergic manipulations.

A few studies have also tested the effect of motivational manipulations on temporal bisection performance. For instance, Ward and Odum $(2006,2007)$ reported that choice functions became flatter following pre-feeding, which was interpreted in terms of loss of stimulus control on choice behavior. McClure et al. (2009), on the other hand, reported that extinction and pre-feeding shifted the psychometric function rightward. This observation is more readily attributable to the clock speed effects. More recently, Galtress and Kirkpatrick (2010) first trained rats on a typical temporal bisection task with equal reward for the correct categorization of the short and long reference durations. In the second phase of their experiment, for one group of rats they increased the reward associated with the correct categorization of the long reference duration to four pellets, whereas for the other group of rats they increased the reward associated with the correct categorization of the short reference duration to four pellets. They observed that reward magnitude manipulation flattened the psychometric functions in both conditions. They also observed that there was a leftward shift in the PSE in the 1-4 pellet condition without any change in the 4-1 pellet condition. In another experiment, Galtress and Kirkpatrick (2010) ruled out satiety as a possible mechanism underlying these effects.

Two major findings emerge from these temporal discrimination studies: (1) Reward magnitude manipulations shifted the PSEs (Galtress \& Kirkpatrick, 2010; McClure et al., 2009); (2) reward magnitude manipulations flattened the psychometric functions, which was typically interpreted in terms of the disruptive effect of within-session and between-session reward magnitude manipulations on the temporal/stimulus control over choice behavior. These primary findings can be interpreted based on the arguments formulated in this paper.

The study by Galtress and Kirkpatrick (2010) can be evaluated within a decision theoretic framework. To that end, both Jozefowiez et al. (2010) and a statistical decision theoretic approach (e.g., Çoşkun et al., in press) predict that subjects' choice functions should shift leftward in the 1-4 pellet condition and rightward in the 4-1 
pellet condition; however, only the former prediction of these approaches was supported by the empirical data. On the other hand, the temporal bisection task can also be treated as a dual PI task, which could in turn lead to different predictions. Machado and Keen's (2003) findings provide empirical support for this approach. They tested pigeons in the temporal bisection task using a long box apparatus and observed that with the onset of the timing stimulus animals moved to the response option associated with the short reference duration and stayed there until the short duration elapsed without reinforcement (e.g., in the long trials). From this point onward, animals moved to the response option associated with the long reference duration and stayed on that side until the end of the timing stimulus. The same profile emerged in the switch task (e.g., Balcı et al., 2008, 2009a). Recently, Maggi et al. (2014) have clearly demonstrated the dual PI-like response pattern in the switch task by introducing probe trials to this task (see their Figs 1 and 3).

One can evaluate the two baseline peak response curves in Fig. 4 within this framework: one curve for the short reference duration (i.e., 1000 arbitrary time units) and the second corresponding curve for the long reference duration (i.e., 2000 arbitrary time units) of the temporal bisection task. The higher likelihood of an animal's occupation of a particular location in the operant box would simply increase the likelihood of the same animal to emit the particular response found at that location.

Under this rationale, the effect of any manipulation on start or stop times of anticipatory responses targeting short and long reference durations would be manifested in terms of the location of the PSE. For instance, assume that subjects started responding earlier for both target intervals; this would result in an earlier switch to the long location and consequently earlier PSE. If, on the other hand, participants started responding later, this would result in a later switch to the long location and consequently a later PSE. Note that these examples assume that stop times of responses for the short reference duration are constrained by the start times of responses for the long reference duration. Galtress and Kirkpatrick's (2010) findings (earlier PSE in the 1-4 pellet condition with no effect in the 4-1 pellet condition after baseline training with a 1-1 pellet condition) are consistent with this reasoning. In the 1-4 pellet condition, the reward magnitude manipulation would be expected to affect only the start time of the responses for the long reference duration (and thus leading to earlier PSE), whereas in the 4-1 pellet condition reward magnitude manipulation would affect the start time of the responses targeting the short reference duration (and thus not exerting any notable effect on the PSE).

In order to better demonstrate these predictions, the normalized response rates (normalized by the maximum response rate of the corresponding FI) for the short reference duration (i.e., 1000 arbitrary time units) were subtracted from the normalized response rates for the long reference duration (i.e., 2000 arbitrary time units) at different time points in a trial (and then these values were transformed by adding 1 and dividing by 2 to fit the difference score in the $0-1$ range). Figure 5 

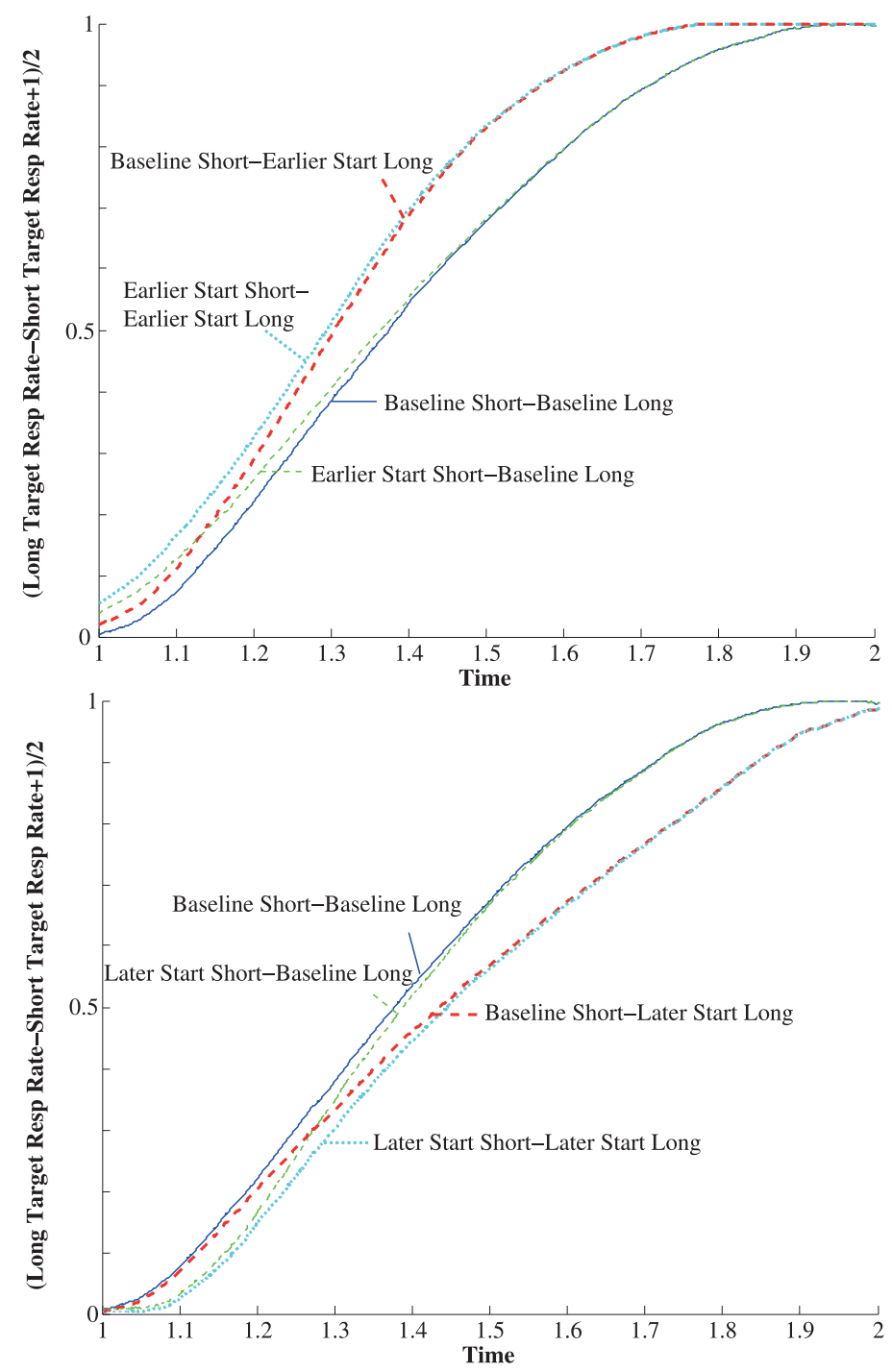

Figure 5. Predicted psychometric functions when the temporal bisection task is treated as a dual PI procedure and differences between response rates for two different target durations are computed as described in the main text. Top and bottom panels show that earlier and later start times for long reference duration lead to leftward and rightward shifts in the psychometric function, respectively. Start time modulation for the short target duration does not lead to any shifts in the psychometric function. Consequently, this view accounts for the results of Galtress and Kirkpatrick (2010). This figure is published in color in the online version. 
shows the predicted psychometric functions for different comparisons (e.g., earlier start for short reference duration and baseline responding for long reference duration).

The results of these computations supported the predictions formulated above: when the temporal bisection task is treated as a dual PI task, earlier or later start times (due to response threshold modulation) for the long reference duration can lead to leftward and rightward shifts in the psychometric function, respectively. This does not hold for responses for the short reference duration.

The other major finding, namely the flatter psychometric functions, can be explained by increased start time/stop time variability or wider response time distributions as a result of motivational manipulations. Unfortunately, these variability indices have not been systematically investigated in the studies that tested the effect of motivational state on peak responding. In one of these studies, Balcı et al. (2013) showed that higher reward magnitudes led to higher stop time CVs, which would be reflected in a shallower psychometric function. Further studies are required to elucidate the source of flattening in choice functions.

\section{Discussion}

In individual peak interval trials, subjects abruptly initiate their anticipatory responses prior to the reward availability time and they abruptly terminate their anticipatory responses if the reward is omitted. On the other hand, the majority of the studies that utilized the PI procedure presented their data in terms of the average peak response curves. Adopting this analytical approach, a large number of studies tested the acute effects of dopamine agonists and antagonists and showed a leftward and rightward shift in peak times, respectively as a result of these manipulations (for a review see Cheng et al., 2007b). These shifts in peak times were typically treated as a proxy for an overall shift in the peak response curves, and thus they have been primarily interpreted in terms of the clock speed modulation within the framework of the dopamine clock hypothesis (e.g., Meck, 2006).

This paper argued that the effect of these critical manipulations should always be evaluated by adopting theory-driven analytical approaches to responding at the level of individual trials (but see limitations below) rather than solely focusing on peak locations of the average response curves (i.e., peak times). This argument is primarily based on the fact that the typical effect of dopaminergic manipulations on peak times is subject to averaging artifacts (see also Saulgiver et al., 2006; Taylor et al., 2007). Specifically, lowering or increasing the decision threshold for response initiation (start threshold) itself can lead to proportional leftward and rightward shifts in the peak times respectively, when the data are averaged across multiple PI trials. On the other hand, since the clock speed effects would be expected to affect the timing of both response initiations and termination in the same direction and 
to the same degree, asymmetrical effects on start and stop times actually constitute an empirical challenge for the clock speed based accounts.

A number of studies that investigated the effect of dopaminergic manipulations (pharmacological and genetic) on peak responding by focusing on responses in individual trials have indeed shown that the effects were primarily on start times in the absence of a change in stop times (for summary, see Table 1). These findings are consistent with the view that start and stop thresholds are independent processes (Gallistel et al., 2004b; Gibbon \& Church, 1990), an approach that accommodates their independent setting and acquisition (Balcr et al., 2009b; MacDonald et al., 2012). Interestingly, the review of the literature on the effect of motivation on peak responding has also revealed a very similar behavioral signature to the one described above. Specifically, higher reward magnitudes led to earlier start times, whereas reward devaluation led to later start times. Importantly, these manipulations typically did not affect the stop times. Given these consistent observations regarding the asymmetrical effects of motivational manipulations on start and stop times and the known role of dopaminergic function for motivation (Berridge, 2007; Wise, 2004), it is possible that the effects of dopaminergic agonists and antagonists in the studies reviewed here are mediated by the motivational state of the animal and that these effects result from the modulation of start thresholds rather than the clock speed.

Previous studies (Cheng et al., 2007a, b) have shown that dopaminergic manipulations lead to typical peak time shifts in animals that have received moderate amount of training ( $<30$ sessions) and disrupted temporal control over anticipatory responding if animals are over trained ( $>180$ sessions). These findings are also consistent with the sensitivity of goal-directed behavior/insensitivity of habitual responses to motivational manipulations (e.g., Adams, 1981; Balleine \& Dickinson, 1998). The level of training might be a factor that also explains the discrepancy between those studies that reported start time $v s$. stop time modulation as result of dopaminergic manipulations. The level of training would be expected to cause shifts in the neural substrates that underlie peak responding. Consequently, the same pharmacological manipulations might affect the timing behavior differently at different points in training (Yin et al., 2004). On the other hand, the comparison between studies that showed an effect on start times $v s$. stop times (see Table 1) did not reveal any apparent differences in terms of the level of training. Future studies are required to elucidate the source of this potentially important discrepancy.

One of the interesting facts is that the effect of motivational manipulations and genetic factors on timing behavior appears to last for many sessions whereas the effect of dopaminergic manipulations have been typically reported to be transient and normalize in chronic dosing regimes (e.g., Meck, 1996). Any effect due to decision threshold setting would indeed be expected to survive chronic manipulations whereas clock speed effects would be expected to be short-lived due to renormalization of the LTM representation. Since the majority of the studies that 
investigated the effect of dopaminergic manipulations did not utilize chronic dosing regimes, we do not know if the effects reported in some of those studies would have lasted longer in chronic conditions. One of the possibilities is that dopaminergic manipulations exert their effect on both clock speed and motivational state independently possibly at different doses. In this case, what experimenters observe would be a joint effect of the drug administration on clock speed and decision thresholds. With extensive training, the clock speed effects would normalize however the behavioral effects due to start threshold modulation would persist. If the start and stop thresholds are both lowered by motivational manipulations (due to single threshold assumption), this would predict that in chronic dosing regimes, the peak time should normalize but the response curve should have a wider spread. These behavioral patterns can indeed be used as model-based benchmarks for the interpretation of future findings.

The shifts in start times in the absence of an effect on stop times are also difficult to address within the behavioral theory of timing (BET) framework (Killeen \& Fetterman, 1988). The BET assumes a chain of behavioral states and that the animal transitions between these states with elapsing time. Importantly, it is assumed that the inter-state transition delays are exponentially distributed and the rate of the exponential process increases with increasing reward rate (e.g., shorter delay to a fixed reward, larger reward after a fixed delay). Since increases in reward magnitude should result in faster transitions between behavioral states (faster Poisson pacemaker), it should result in earlier start and stop times. As noted repeatedly, this does not seem to be the case in the empirical data described in this paper.

A number of studies have shown that dopaminergic manipulations can also alter the temporal characteristics of the instinctive behaviors such as grooming (Matell et al., 2006a), licking (Badiani \& Stewart, 1999), and food protection behavior (Wallace et al., 2006) in the directions predicted by the dopamine clock hypothesis. For instance, amphetamine shifted the inter-lick-interval distributions leftward (Wallace et al., 2006) whereas pimozide had the opposite effect (Gramling et al., 1986). These effects have been interpreted in terms of the clock speed effects and the motivation/threshold-based explanations formulated in this paper cannot readily account for these findings. That said, some of the reported effects in these studies contradict the predictions made about the learned timed behaviors. For instance, Badiani and Stewart (1999) reported sensitization rather than the normalization of inter-lick intervals with chronic dopamine treatments. Furthermore, Matell et al. (2006a) showed the effect of D1 receptor agonist on temporal control over grooming whereas D2 receptor affinity of the drugs have been argued to be important for clock speed effects (e.g., Drew et al., 2003; Meck, 1986). In any case, this paper does not argue that motivational mechanisms are solely responsible for the effects of dopaminergic manipulations on timing processes. Motivational factors might just be an independent source of an influence on timed behavior. Theory 
driven elaborate analyses of the data can differentiate those effects that are driven by motivational factors and clock speed.

So far, I have exercised an approach that was inline with the STT specifically relying on its implicit single clock assumption. On the other hand, the findings discussed in the current manuscript can also be interpreted with alternative theoretical approaches. For instance, instead of relying on a single clock process (e.g., parameterized to hit a single threshold at the fixed interval as argued by Simen et al., 2011, 2013), subjects might be relying on two independent clocks, one parameterized to hit a single threshold at the start time and the other one parameterized to hit the same threshold at the stop time. In this case, the effect of dopamine agonists and antagonists might affect the speed of both clocks. If dopaminergic manipulations would decrease or increase the rate of temporal integration by some constant value, the resultant effect would be more pronounced for stop times compared to start times, which does not fit the results reviewed in this paper. If the dopaminergic manipulation decreased or increased the rate of accumulation by some constant proportion then the resultant effect would be equivalent for both start and stop times, which is also not supported by majority of the findings discussed in this paper. Thus, a two clock based model is not a parsimonious alternative explanation for the observed effects compared to other variations of the STT (e.g., independent thresholds).

Due to the risk of averaging artifacts as demonstrated in a number of studies reviewed in this paper, establishing clock speed effects requires single trial analyses and entails shifts in both start and stop times to the same degree in the same direction. Averaging artifacts are not specific to the PI procedure. Gallistel et al. (2004a) and Papachristos and Gallistel (2006) have nicely demonstrated that averaging (between subjects) might lead to invalid inferences regarding the individual animal's acquisition curve (also see Balcı et al., 2009b; Skinner, 1976). In this sense, the analytical issues discussed in this paper constitute just another example of the dangers of data averaging. This paper has shown that asymmetrical modulation of start and stop thresholds can also account for the several reported effects of motivational and dopaminergic manipulations on the temporal bisection performance, when this procedure is conceptualized as a dual PI task.

The arguments developed in this paper relied primarily on the endpoints gathered from the single-trials analysis of peak responding. On the other hand, this analytical approach has several important limitations in terms of its applicability that need to be kept in mind prior to its use. Here, I will list few of these issues, which were originally reported in Yin et al. (in press):

(1) The orderliness of the average response curves should be the first criterion for applying single trial analysis. Researchers should make sure that the peak of response curve is located around the target duration and that it has a close to symmetrical shape with narrow spread. Thus, this analysis is fit only when temporal control over behavior in manifested. In other cases, the output this analysis might 
not provide meaningful results. (2) There are multiple methods for identifying the start and stop times (e.g., Balcı et al., 2009b; Cheng \& Westwood, 1993; Church et al., 1994; Taylor et al., 2007). Although in essence all of these approaches have been developed to detect state transitions in terms of response rates, they use different computations and rely on different constraints that can result in differences in the output for some datasets. The data should be studied well (e.g., is there a second run period?) prior to determining which method to utilize. (3) A related issue concerns the inclusion criteria adopted for the data analyses. Some studies constrain their analyses to a subset of data based on the location of the start and stop times with respect to the target duration. For instance, start times are preferred to occur prior to whereas stop times are preferred to occur after the target time (e.g., Church et al., 1994). On the other hand, there is no consensus in the literature regarding these criteria and further theoretical studies are needed to resolve these analytical issues. (4) One of the assumptions of single-trials analysis is that the response rate stays constant within each state. On the other hand, sometimes this assumption does not hold in the data (e.g., Meck \& Williams, 1997). (5) Testing animals with short target durations can result in high response rates with the onset of the CS possibly due to the high associative strength of the CS. This can be treated as another case of loss of temporal control constraining the applicability of the single-trials analysis as discussed under issue number 1 . These issues/cautions should be exercised and interpreted in relation to the data at hand before applying these more sophisticated approaches (for a more extensive list of related limitations see Yin et al., in press).

This review overall suggested a possible motivation-based explanation for the typical dopamine-dependent peak time shifts with a specific emphasis on analytical approaches. I emphasize that this account does not nullify the possible independent clock speed effects but it rather warns against possible misleading conclusions based on the choice of analytical approaches. These issues can guide future research and where appropriate motivate the use of theory-driven elaborate analytical methods for the psycho-mechanistic interpretation of the behavioral findings. The dopamine clock hypothesis remains an influential model in the field and continues to guide further research on the neurobiological basis of interval timing.

\section{Acknowledgements}

I would like to thank Warren H. Meck for formulating the limitations of the applicability of the single-trials analyses. This work was supported by the FP7 Marie Curie PIRG08-GA-2010-277015, TUBITAK 1001 \#111K402, and BAGEP Grant from Bilim Akademisi — The Science Academy, Turkey to Fuat Balcı. 


\section{References}

Abner, R. T., Edwards, T., Douglas, A., \& Brunner, D. (2001). Pharmacology of temporal cognition in two mouse strains. Int.J. Comp. Psychol., 14, 189-210.

Adams, C. D. (1981). Variations in the sensitivity of instrumental responding to reinforcer devaluation. Q.J. Exp. Psychol. B, 34, 77-98.

Badiani, A., \& Stewart, J. (1999). Long-lasting sensitization to the accelerating effects of amphetamine on the speed of an internal clock. Behav. Brain Res., 100, 217-223.

Balcı, F., Papachristos, E. B., Gallistel, C. R., Brunner, D., Gibson, J., \& Shumyatsky, G. P. (2008). Intervaltiming in the genetically modified mouse: A simple paradigm. Genes, Brain Behav., 7, 373-384.

Balcı, F., Freestone, D., \& Gallistel, C. R. (2009a). Risk assessment in man and mouse. Proc. Natl Acad. Sci. USA, 106, 2459-2463.

Balcı, F., Gallistel, C. R., Allen, B. D., Frank, K., Gibson, J., \& Brunner, D. (2009b). Acquisition of peak responding: What is learned? Behav. Proc., 80, 67-75.

Balcı, F., Ludvig, E. A., Abner, R., Zhuang, X., Poon, P., \& Brunner, D. (2010a). Motivational effects on interval timing in dopamine transporter (DAT) knock-down mice. Brain Res., 1325, 89-99.

Balcı, F., Ludvig, E. A., \& Brunner, D. (2010b). Within-session modulation of timed anticipatory responding: When to start responding. Behav. Proc., 85, 204-206.

Balcı, F., Wiener, M., Cavdaroglu, B., \& Coslett, B. H. (2013). Epistasis effects of dopamine genes on interval timing and reward magnitude in humans. Neuropsychologia, 51, 293-308.

Balleine, B. W., \& Dickinson, A. (1998). Goal-directed instrumental action: Contingency and incentive learning and their cortical substrates. Neuropharmacology, 37, 407-419.

Berridge, K. C. (2007). The debate over dopamine's role in reward: The case for incentive salience. Psychopharmacology, 191, 391-431.

Berridge, K. C., \& Robinson, T. E. (1998). What is the role of dopamine in reward: Hedonic impact, reward learning, or incentive salience? Brain Res. Rev., 28, 309-369.

Bizot, J. C. (1997). Effects of psychoactive drugs on temporal discrimination in rats. Behav. Pharmacol., 8, 293-308.

Buhusi, C. V., \& Meck, W. H. (2005). What makes us tick? Functional and neural mechanisms of interval timing. Nat. Rev. Neurosci., 6, 755-765.

Buhusi, C. V., Aziz, D., Winslow, D., Carter, R. E., Swearingen, J. E., \& Buhusi, M. C. (2009). Interval timing accuracy and scalar timing in C57BL/6 mice. Behav. Neurosci., 123, 1102-1113.

Cagniard, B., Balsam, P. D., Brunner, D., \& Zhuang, X. (2006). Mice with chronically elevated dopamine exhibit enhanced motivation, but not learning, for a food reward. Neuropsychopharmacology, 31, 1362-1370.

Catania, A. C. (1970). Reinforcement schedules and psychophysical judgements: A study of some temporal properties of behavior. In W. N. Schoenfeld (Ed.), The theory of reinforcement schedules (pp. 1-42). New York, NY, USA: Appleton-Century-Croft.

Çevik, M. O. (2003). Effects of methamphetamine on duration discrimination. Behav. Neurosci., 117, 774-784.

Cheng, K., \& Westwood, R. (1993). Analysis of single trials in pigeons timing performance.J. Exp. Psychol. Anim. Behav. Process., 19, 56-67.

Cheng, R. K., Ali, Y. M., \& Meck, W. H. (2007a). Ketamine 'unlocks' the reduced clock-speed effect of cocaine following extended training: Evidence for dopamine-glutamate interactions in timing and time perception. Neurobiol. Learn. Mem., 88, 149-159. 
Cheng, R. K., Hakak, O. L., \& Meck, W. H. (2007b). Habit formation and the loss of control of an internal clock: Inverse relationship between the level of baseline training and the clock-speed enhancing effects of methamphetamine. Psychopharmacology (Berl.), 193, 351-362.

Chiang, T.-J., Al-Ruwaitea, A. S. A., Mobini, S., Ho, M.-Y., Bradshaw, C. M., \& Szabadi, E. (2000). The effect of D-amphetamine on performance on two operant timing schedules. Psychopharmacology, $150,170-184$.

Church, R. M., Miller, K. D., Meck, W. H., \& Gibbon, J. (1991). Symmetrical and asymmetrical sources of variance in temporal generalization. Anim. Learn. Behav., 19, 207-214.

Church, R. M., Meck, W. H., \& Gibbon, J. (1994). Application of scalar timing theory to individual trials. J. Exp. Psychol. Anim. Behav. Process., 20, 135-155.

Church, R. M., Lacourse, D. M., \& Crystal, J. D. (1998). Temporal search as a function of the variability of interfood intervals. J. Exp. Psychol. Anim. Behav. Process., 24, 291-315.

Çoşkun, F., Sayalı, C., Gürbüz, E., \& Balcı, F. (in press). Optimal temporal discrimination. Q. J. Exp. Psychol. doi:10.1080/17470218.2014.944921.

Coull, J. T., Cheng, R. K., \& Meck, W. H. (2011). Neuroanatomical and neurochemical substrates of timing. Neuropsychopharmacology, 36, 3-25.

Doughty, A. H., \& Richards, J. B. (2002). Effects of reinforcer magnitude on responding under differential-reinforcement-of-low-rate schedules of rats and pigeons. J. Exp. Anal. Behav., 78, 1730.

Drew, M. R., Fairhurst, S., Malapani, C., Horvitz, J. C., \& Balsam, P. D. (2003). Effects of dopamine antagonists on the timing of two intervals. Pharmacol. Biochem. Behav., 75, 9-15.

Fox, A. E., \& Kyonka, E. G. (2014). Choice and timing in pigeons under differing levels of food deprivation. Behav. Proc., 106, 82-90.

Gallistel, C. R., Balsam, P. D., \& Fairhurst, S. (2004a). The learning curve: Implications of a quantitative analysis. Proc. Natl Acad. Sci. USA, 101, 13124-13131.

Gallistel, C. R., King, A., \& McDonald, R. (2004b). Sources of variability and systematic error in mouse timing behavior.J. Exp. Psychol. Anim. Behav. Process., 30, 3-16.

Galtress, T., \& Kirkpatrick, K. (2009). Reward magnitude effects on temporal discrimination. Learn. Motiv., 41, 108-124.

Galtress, T., \& Kirkpatrick, K. (2010). The role of the nucleus accumbens core in impulsive choice, timing, and reward processing. Behav. Neurosci., 124, 26-43.

Galtress, T., Garcia, A., \& Kirkpatrick, K. (2012a). Individual differences in impulsive choice and timing in rats. J. Exp. Anal. Behav., 98, 65-87.

Galtress, T., Marshall, A. T., \& Kirkpatrick, K. (2012b). Motivation and timing: Clues for modeling the reward system. Behav. Proc., 90, 142-153.

Gershman, S.J., Moustafa, A. A., \& Ludvig, E. A. (2014). Time representation in reinforcement learning models of the basal ganglia. Front. Comput. Neurosci., 7, 194. doi:10.3389/fncom.2013.00194.

Gibbon, J. (1977). Scalar expectancy theory and Weber's Law in animal timing. Psychol. Rev., 84, 279325.

Gibbon, J., Church, R. M., \& Meck, W. H. (1984). Scalar timing in memory. In J. Gibbon \& L. Allan(Eds.), Timing and time perception (pp. 52-77). New York, NY, USA: New York Academy of Sciences.

Gibbon, J., \& Church, R. M. (1990). Representation of time. Cognition, 37, 23-54.

Gibbon, J., \& Church, R. M. (1992). Comparison of variance and covariance patterns in parallel and serial theories of timing.J. Exp. Anal. Behav., 57, 393-406.

Gooch, C., Wiener, M., Portugal, G., \& Matell, M. (2007). Evidence for separate neural mechanisms for the timing of discrete and sustained responses. Brain Res., 1156, 139-151. 
Grace, R. C., \& Nevin, J. A. (2000). Response strength and temporal control in fixed interval schedules. Anim. Learn. Behav., 28, 313-331.

Gramling, S. E., \& Fowler, S. C. (1986). Some effects of pimozide and of shifts in sucrose concentration on lick rate, duration, and interlick interval. Pharmacol. Biochem. Behav., 25, 219-222.

Hatten, J. L., \& Shull, R. L. (1983). Pausing on fixed-interval schedules: Effects of the prior feeder duration. Behav. Anal. Lett., 3, 101-111.

Jozefowiez, J., Staddon, J. E., \& Cerutti, D. T. (2010). The behavioral economics of choice and interval timing. Psychol. Rev., 116, 519-539.

Kacelnik, A., \& Brunner, D. (2002). Timing and foraging: Gibbon's scalar expectancy theory and optimal patch exploitation. Learn. Motiv., 33, 177-195.

Killeen, P., \& Fetterman, J. G. (1988). A behavioral theory of timing. Psychol. Rev., 95, 274-295.

Lake, J. I., \& Meck, W. H. (2013). Differential effects of amphetamine and haloperidol on temporal reproduction: Dopaminergic regulation of attention and clock speed. Neuropsychologia, 51, 284292.

Ludvig, E., Conover, K., \& Shizgal, P. (2007). The effects of reinforcer magnitude on timing in rats. J. Exp. Anal. Behav., 87, 201-218.

Ludvig, E. A., Balci, F., \& Spetch, M. L. (2011). Reward magnitude and timing in pigeons. Behav. Proc., 86, 359-363.

MacDonald, C. J., Cheng, R. K., \& Meck, W. H. (2012). Acquisition of 'Start' and 'Stop' response thresholds in peak-interval timing is differentially sensitive to protein synthesis inhibition in the dorsal and ventral striatum. Front. Integr. Neurosci., 6, 10. doi:10.3389/fnint.2012.00010.

MacEwen, D., \& Killeen, P. (1991). The effects of rate and amount of reinforcement on the speed of the pacemaker in pigeons' timing behavior. Anim. Learn. Behav., 19, 164-170.

Machado, A., \& Keen, R. (2003). Temporal discrimination in a long operant chamber. Behav. Proc., $62,157-182$.

Maggi, D., Garbugino, L., Heise, I., Nieus, T., Balci, F., Wells, S., Tocchini-Valentini, G. P., Mandillo, S., Nolan, P. M., \& Tucci, V. (2014). A cross-laboratory investigation of timing endophenotypes in mouse behavior. Timing Time Percept., 2, 35-40.

Malapani, C., Deweer, B., \& Gibbon, J. (2002). Separating storage from retrieval dysfunction of temporal memory in Parkinson's disease.J. Cogn. Neurosci., 14, 311-322.

Malapani, C., \& Fairhurst, S. (2002). Scalar timing in animals and humans. Learn. Motiv., 33, 156-176.

Malapani, C., \& Rakitin, B. C. (2003). Interval timing in the dopamine-depleted basal ganglia: From empirical data to timing theory. In W. H. Meck (Ed.), Functional and neural mechanisms of interval timing (pp. 485-514). Boca Raton, FL, USA: CRC Press.

Maricq, A. V., \& Church, R. M. (1983). The differential effects of haloperidol and methamphetamine on time estimation in the rat. Psychopharmacology (Berl.), 79, 10-15.

Maricq, A. V., Roberts, S., \& Church, R. M. (1981). Methamphetamine and time estimation.J. Exp. Psychol. Anim. Behav. Process., 7, 18-30.

Mattell, M. S., King, R. K., \& Meck, W. H. (2004). Differential modulation of clock speed by the administration of intermittent versus continuous cocaine. Behav. Neurosci., 118(1), 150-156.

Matell, M. S., Aldridge, J. W., \& Berridge, K. C. (2006a). Dopamine D1 activation shortens the duration of phases in stereotyped grooming sequences. Behav. Proc., 71, 241-249.

Matell, M. S., Bateman, M., \& Meck, W. H. (2006b). Single-trials analyses demonstrate that increases in clock speed contribute to the methamphetamine-induced horizontal shifts in peak-interval timing functions. Psychopharmacology, 188, 201-212. 
McClure, E. A., Saulsgiver, K. A., \& Wynne, C. D. L. (2005). Effects of D-amphetamine on temporal discrimination in pigeons. Behav. Pharmacol., 16, 193-208.

McClure, E. A., Saulsgiver, K. A., \& Wynne, C. D. L. (2009). Manipulating pre-feed, density of reinforcement, and extinction produces disruption in the location variation of a temporal discrimination task in pigeons. Behav. Proc., 82, 85-89.

Meck, W. H. (1983). Selective adjustment of the speed of internal clock and memory processes. J. Exp. Psychol. Anim. Behav. Process., 9, 171-201.

Meck, W. H. (1986). Affinity for the dopamine D2 receptor predicts neuroleptic potency in decreasing the speed of an internal clock. Pharmacol. Biochem. Behav., 25, 1185-1189.

Meck, W. H. (1988). Internal clock and reward pathways share physiologically similar informationprocessing stages. In M. L. Commons, R. M. Church, J. R. Stellar, \& A. R. Wagner (Eds.), Quantitative analyses of behavior: Biological determinants of reinforcement (pp. 121-138). Hillsdale, NJ, USA: Erlbaum.

Meck, W. H. (1996). Neuropharmacology of timing and time perception. Cogn. Brain Res., 3, 227-242.

Meck, W. H. (2006). Neuroanatomical localization of an internal clock: A functional link between mesolimbic, nigrostriatal, and mesocortical dopaminergic systems. Brain Res., 1109, 93-107.

Meck, W. H., \& Williams, C. L. (1997). Characterization of the facilitative effects of perinatal choline supplementation on timing and temporal memory. Neuroreport, 8, 2831-2835.

Odum, A. L., Lieving, L. M., \& Schaal, D. W. (2002). Effects of D-amphetamine in a temporal discrimination procedure: Selective changes in timing or rate dependency? J. Exp. Anal. Behav., 78, 195-214.

Odum, A. L., \& Ward, R. D. (2007). Characterizing the effects of D-amphetamine on temporal discrimination. Behav. Proc., 75, 156-166.

Papachristos, E. B., \& Gallistel, C. R. (2006). Autoshaped head poking in the mouse: A quantitative analysis of the learning curve. J. Exp. Anal. Behav., 85, 293-308.

Paterson, N. E., Balci, F., Campbell, U., Olivier, B., \& Hanania, T. (2011). The triple reuptake inhibitor DOV216,303 exhibits limited antidepressant-like properties in the differential reinforcement of low-rate 72-sec responding assay, likely due to dopamine reuptake inhibition.J. Psychopharmacol., 25, 1357-1364.

Peciña, S., Cagniard, B., Berridge, K. C., Aldridge, J. W., \& Zhuang, X. (2003). Hyperdopaminergic mutant mice have higher 'wanting' but not 'liking' for sweet rewards. J. Neurosci., 23, 9395-9402.

Penney, T. B., Gibbon, J., \& Meck, W. H. (2008). Categorical scaling of duration bisection in pigeons (Columba livia), mice (Mus musculus), and humans (Homo sapiens). Psychol. Sci., 19, 1102-1108.

Plowright, C. M. S., Church, D., Behnke, P., \& Silverman, A. (2000). Time estimation by pigeons on a fixed interval: The effect of pre-feeding. Behav. Proc., 52, 43-48.

Rakitin, B. C., Gibbon, J., Penney, T. B., Malapani, C., Hinton, S. C., \& Meck, W. H. (1998). Scalar expectancy theory and peak-interval timing in humans. J. Exp. Anal. Behav., 24, 15-33.

Rammsayer, T. (1989). Dopaminergic and serotoninergic influence on duration discrimination and vigilance. Pharmacopsychiatry, 22(Suppl. 1), 39-43.

Rammsayer, T. H. (1993). On dopaminergic modulation of temporal information processing. Biol. Psychol., 36, 209-222.

Rammsayer, T. H. (1997). Are there dissociable roles of the mesostriatal and mesolimbocortical dopamine systems on temporal information processing in humans? Neuropsychobiology, 35, 36 45.

Rammsayer, T. H., \& Vogel, W. H. (1992). Pharmacologic properties of the internal clock underlying time perception in humans. Neuropsychobiology, 26, 71-80. 
Reuter, M., Schmitz, A., Corr, P., \& Hennig, J. (2006). Molecular genetics support Gray's personality theory: The interaction of COMT and DRD2 polymorphisms predicts the behavioural approach system. Int. J. Neuropsychopharmacol., 9, 155-166.

Roberts, S. (1981). Isolation of an internal clock.J. Exp. Psychol. Anim. Behav. Process., 7, 242-268.

Santi, A., Weise, L., \& Kuiper, D. (1995). Amphetamine and memory for event duration in rats and pigeons: Disruption of attention to temporal samples rather than changes in the speed of the internal clock. Psychobiology, 23, 224-232.

Saulsgiver, K. A., McClure, E. A., \& Wynne, C. D. (2006). Effects of D-amphetamine on the behavior of pigeons exposed to the peak procedure. Behav. Proc., 71, 268-285.

Schneider, B. A. (1969). A two-state analysis of fixed-interval responding in the pigeon. J. Exp. Anal. Behav., 12, 677-687.

Simen, P., Balcı, F., deSouza, L., Holmes, P., \& Cohen, J. D. (2011). A model of interval timing by neural integration. J. Neurosci., 31, 9238-9253.

Simen, P., Rivest, F., Ludvig, E. A., Balci, F., \& Killeen, P. (2013). Timescale invariance in the pacemakeraccumulator family of timing models. Timing Time Percept., 30, 159-188.

Skinner, B. F. (1976). Farewell, my lovely! J. Exp. Anal. Behav., 25, 218.

Taylor, K. M., Horvitz, J. C., \& Balsam, P. D. (2007). Amphetamine affects the start of responding in the peak interval timing task. Behav. Proc., 74, 168-175.

Wallace, D. G., Wallace, P. S., Field, E., \& Whishaw, I. Q. (2006). Pharmacological manipulations of food protection behavior in rats: Evidence for dopaminergic contributions to time perception during a natural behavior. Brain Res., 1112, 213-221.

Ward, R. D., \& Odum, A. L. (2006). Effects of prefeeding, intercomponent-interval food, and extinction on temporal discrimination and pacemaker rate. Behav. Proc., 71, 297-306.

Ward, R. D., \& Odum, A. L. (2007). Disruption of temporal discrimination and the choose-short effect. Learn. Behav., 35, 60-70.

Wise, R. A. (2004). Dopamine, learning and motivation. Nat. Rev. Neurosci., 5, 483-494.

Yi, L. (2007). Applications of timing theories to a peak procedure. Behav. Proc., 75, 188-198.

Yin, B., Lusk, N. A., \& Meck, W. H. (in press). Interval-timing protocols and their relevancy to the study of temporal cognition and neurobehavioral genetics. In V. Tucci (Ed.), Neuro-phenome: Cuttingedge approaches and technologies in neurobehavioral genetics. Wiley-Blackwell.

Yin, H. H., Knowlton, B. J., \& Balleine, B. W. (2004). Lesions of dorsolateral striatum preserve outcome expectancy but disrupt habit formation in instrumental learning. Eur. J. Neurosci., 19, 181-189.

Yin, H. H., Zhuang, X., \& Balleine, B. W. (2006). Instrumental learning in hyperdopaminergic mice. Neurobiol. Learn. Mem., 85, 283-288.

Zhuang, X., Oosting, R. S., Jones, S. R., Gainetdinov, R. R., Miller, G. W., Caron, M. G., \& Hen, R. (2001). Hyperactivity and impaired response habituation in hyperdopaminergic mice. Proc. Natl Acad. Sci. USA, 98, 1982-1987. 